\title{
Research Progress of Anti-Tumor Angiogenesis in Traditional Chinese Medicine
}

\author{
Tao Gou ${ }^{*}$, Hailong $\mathrm{Si}^{2}$ \\ ${ }^{1}$ Shaanxi University of Chinese Medicine, Xianyang 712000, Shaanxi Province, China \\ ${ }^{2}$ The Affiliated Hospital of Shaanxi University of Chinese Medicine, Xianyang 712000, Shaanxi Province, China \\ *Corresponding author: Tao Gou, $1203274968 @ q q . c o m$
}

\begin{abstract}
In recent years, seeking effective anti-angiogenic components and therapeutic methods from traditional Chinese Medicine (TCM) has been a hot spot in the treatment of malignant tumors. In particular, the active ingredients found in Chinese herbal extracts have shown strong inhibition of tumor neovascularization.
\end{abstract}

Keywords: Traditional Chinese medicine; Tumor; Anti-angiogenic; Overview

Publication date: November 2021; Online publication: November 30, 2021

\section{Tumor and angiogenesis}

In 1971, Folkman proposed a hypothesis, in which angiogenesis is required for the development and growth of solid tumors larger than 1-2 $\mathrm{mm}{ }^{[1]}$. Thereafter, evidence showed that solid tumors depend on the formation of new blood vessels in order to continue to grow ${ }^{[2]}$. Inspired by this, anti-angiogenic strategies began to become the focus of research teams, in which these strategies may develop into new treatment methods for solid tumors. Over 40 years of research, researchers have accumulated a large amount of data, supporting Folkman's hypothesis ${ }^{[3]}$. Relying on the efforts in this field of research, the complex mechanism of tumor angiogenesis has been exposed. The normal process of angiogenesis is in a relatively dynamic steady state and is strictly controlled by pro-angiogenic and anti-angiogenic regulators. Once this homeostasis is destroyed, the finger phenotypic "angiogenic switch" becomes active and triggers angiogenesis ${ }^{[4]}$. Hypoxia is one of the main factors driving tumor angiogenesis, resulting in increased expression of vascular endothelial growth factors and other angiogenesis stimulating factors in hypoxic cells ${ }^{[5]}$.

\section{The understanding of tumor angiogenesis in traditional Chinese medicine}

Traditional Chinese medicine (TCM) is one of the important means to prevent and treat tumors in China. The concept of "cancer virus" as the specific pathogenic factor of tumors has become the consensus of many scholars. It is also the premise of tumor angiogenesis ${ }^{[6]}$. The theory of oncogenesis caused by "cancer toxin" was first put forward by Professor Zhongying Zhou, a master of Chinese medicine. He believes that cancer toxin is one of the toxic evils and claims that it is a specific disease that leads to the occurrence and development of tumors on the basis of internal and external factors acting on the body, leading to visceral dysfunction, or induced by internal and external factors on the basis of visceral dysfunction ${ }^{[7]}$. Correspondingly, a single tumor cell is not enough to form a tumor. Any tumor consists of tumor parenchyma and tumor stroma; tumor cells are components of tumor parenchyma, while tumor stroma refers to connective tissues, including blood vessels and lymphatic vessels. The complex interaction 
between them leads to the occurrence of tumor. It can be appreciated that the pathogenesis of cancer in TCM is highly consistent with the understanding of tumor pathogenesis in western medicine.

\section{Effect of traditional Chinese medicine on tumor angiogenesis: Studies on the effective components of single traditional Chinese medicine}

\subsection{Rhizoma Curcumae longae}

Curcumin is a phenolic pigment and a plant polyphenol extracted from Rhizoma Curcumae longae, which has a variety of pharmacological activities. In a research ${ }^{[8]}$, flow cytometry was used to detect the effects of $5-160 \mu \mathrm{mol} / \mathrm{L}$ curcumin on the apoptosis of human umbilical vein endothelial cells (HUVECs). The results showed that the apoptosis rates of HUVECs were 5.5\% 29.8\% after 24 hours of curcumin treatment, which was significantly different from the control group (2.1\%). Gururaj and other researchers found that curcumin reduces the number of HUVECs, not because curcumin is toxic to these cells, but it induces cell apoptosis ${ }^{[9]}$. At present, experimental studies in vivo and in vitro have shown that the anti-angiogenic effect of curcumin is closely related to angiogenic factors.

\subsection{Berberine}

Berberine, also known as berberinum, accounts for about $40 \%$ of the total alkaloids of Coptis chinensis. It belongs to quaternary ammonium isoquinoline alkaloids. It is the main alkaloid of Coptis chinensis, with a content of $5 \% \sim 8 \%$. Berberine is the main effective component of Coptis chinensis ${ }^{[10]}$. Modern pharmacology has proved that berberine has a wide range of pharmacological effects. The literature on berberine as an antitumor drug has a lot of pharmacognostic and clinical evidence. Research has shown that berberine can inhibit tumor cell proliferation by inducing programmed cell death, inhibiting genetic information synthesis, and blocking cell cycle ${ }^{[11]}$. It has potential application prospects in tumor prevention and treatment. In a transplanted tumor model, after acting on human glioblastoma, berberine has a strong inhibitory effect on vascular endothelial cells in vitro. It reduces hemoglobin content and tumor vascular density as well as significantly downregulates the expression of vascular endothelial cell marker CD31. Its mechanism may be realized by regulating VEGFR2/ERK signaling pathway ${ }^{[12]}$.

\subsection{Leech and cantharis}

Modern pharmacological research has shown that the medicinal ingredients of leeches mainly include hirudin, amino acids, phospholipids, glycolipid compounds, sterols, and trace elements, among which hirudin is the main active ingredient. Leech and cantharis can reduce the microvessel density of sarcoma tissues and inhibit the proliferation of vascular endothelial cells; in addition, cantharis has a significant inhibitory effect on angiogenesis. Therefore, one of the anti-tumor effects of leech and cantharis may be achieved by inhibiting angiogenesis with leech and cantharis.

\subsection{Venenum Bufonis}

Bufalin is an anti-cancer active ingredient extracted from Venenum Bufonis. Junshan Liu and other researchers found that arenobufagin can significantly inhibit angiogenesis in vivo and in vitro; they speculated that its inhibitory effect may be related to the induction of vascular endothelial cell cycle arrest and apoptosis ${ }^{[13]}$. Subsequently, another research further pointed out that arenobufagin can inhibit vascular endothelial growth factor-mediated angiogenesis by inhibiting VEGFR-2 signaling pathway ${ }^{[14]}$. Lee and other researchers observed the effect of bufalin on angiogenesis through a capillary-like network structure model generated by primary bovine aortic endothelial cells cultured in three-dimensional type I collagen 
medium. Flow cytometry (FCM) analysis showed that the vascular endothelial cells were blocked in G/M phase and their proliferation was inhibited. The results revealed that $5 \mathrm{nmol} / \mathrm{L}$ of bufalin can significantly inhibit angiogenesis ${ }^{[15]}$. Therefore, the anti-tumor effect of bufalin is closely related to its inhibition of tumor vessel formation.

\section{Problems and prospects}

Tumor angiogenesis is closely related to the formation and prognosis of most tumors. The inhibition of tumor angiogenesis has now become a hot research topic in anti-tumor research. Anti-tumor angiogenesis is not only to inhibit the growth and metastasis of tumor cells, but also to indirectly destroy existing tumor cells. The anti-angiogenesis method of traditional Chinese medicine to treat tumors has opened up new ideas and new ways for Chinese medicine to study about anti-tumor. From the perspective of traditional Chinese medicine theory, there are only a few relevant discussions, and it lacks research on the combined application with other anti-tumor methods in treatment; hence, there is still unicity in treatment. Through syndrome differentiation and treatment of traditional Chinese medicine as well as the use of traditional Chinese medicine theory to guide prescription, the effect of traditional Chinese medicine on inhibiting tumor angiogenesis should be emphasized.

\section{Disclosure statement}

The authors declare that there is no conflict of interest.

\section{References}

[1] Folkman J, 1971, Tumor Angiogenesis: Therapeutic Implications. The New England Journal of Medicine, 285(21): 1182-1186.

[2] Folkman J, 1972, Anti-Angiogenesis: New Concept for Therapy of Solid Tumors. Annals of Surgery, 175(3): 409-416.

[3] Dimova I, Popivanov G, Djonov V, 2014, Angiogenesis in Cancer - General Pathways and Their Therapeutic Implications. Journal of BUON: Official Journal of the Balkan Union of Oncology, 19(1): 15-21.

[4] Ribatti D, Nico B, Crivellato E, et al., 2007, The History of the Angiogenic Switch Concept. Leukemia, 21(1): 44-52.

[5] Dor Y, Porat R, Keshet E, 2001, Vascular Endothelial Growth Factor and Vascular Adjustments to Perturbations in Oxygen Homeostasis. American Journal of Physiology Cell Physiology, 280(6): 13671374.

[6] Zhang T, Sun X, 2016, Mind and Brain Government Apirit: Understanding the Integration of Mind and Brain. Chinese Journal of Rehabilitation Theory and Practice, 22(003): 368-370.

[7] Cheng H, 2014, Discussion on the Theory of Cancer Virus and Pathogenesis. Journal of Traditional Chinese Medicine, 55(20): 1711-1714.

[8] Tie M, Zhang Y, 2014, Discussion on the Mechanism of Promoting Blood Circulation and Removing Stasis to Normalize Tumor Vessels. West China Medical Journal, (12): 2379-2381.

[9] Zheng W, Yang X, Wu L, et al., 2007, Discussion on the Molecular Mechanism of Curcumin Against Angiogenesis. Chinese Journal of Clinical Oncology, 34(12): 683-685.

[10] Gururaj AE, Belakavadi M, Venkatesh DA, et al., 2002, Molecular Mechanisms of Anti-Angiogenic Effect of Curcumin. Biochemical \& Biophysical Research Communications, 297(4): 934-942. 
[11] Ortiz LM, Lombardi P, Tillhon M, et al., 2014, Berberine, an Epiphany Against Cancer. Molecules, 19(8): 12349-12367.

[12] Jin F, Xie T, Huang X, et al., 2018, Berberine Inhibits Angiogenesis in Glioblastoma Xenografts by Targeting the VEGFR2/ERK Pathway. Pharmaceutical Biology, 56(1): 665-71.

[13] Liu J, Zhang D, Chen M, et al., 2011, Inhibition of Angiogenesis by Arenobufagin. Acta Pharmaceutica Sinica, (05): 527-533.

[14] Li M, Wu S, Liu Z, et al., 2012, Arenobufagin, a Bufadienolide Compound from Toad Venom, Inhibits Vegf-Mediated Angiogenesis Through Suppression of Vegfr-2 Signaling Pathway. Biochemical Pharmacology, 83(9): 1251-1260.

[15] Lee DY, Yasuda M, Yamamoto T, et al., 1997, Bufalin Inhibits Endothelial Cell Proliferation and Angiogenesis In Vitro. Life Sciences, 60(2): 127-134. 